\title{
Cooperación académica entre Corea del Sur y América Latina: el Programa e-School de la Fundación Corea y el uso de las Tecnologías de la Información y Comunicación para la internacionalización educativa
}

\author{
JuAn Felipe López Aymes * \\ RENATO BALDERRAMA SANTANDER** \\ ANA Karen VelÁZQUEZ***
}

*CRIM-UNAM
${ }^{* *}$ CEA-UANL
$* * *$ CEA-UANL

Este reporte fue realizado gracias al financiamiento de la Red sobre Internacionalización y Movilidades Académicas y Científicas (RIMAC) Proyecto 293325 del Programa de Redes Temáticas, CONACYT, México.

\begin{abstract}
Resumen
En este artículo se presenta la implementación, restructura y evaluación del Programa e-School para América Latina auspiciado por la Fundación Corea para impulsar los estudios asiáticos y coreanistas en la región. Aquí se apunta al cambio de sede de la coordinación de California a Nuevo León como fundamental para el crecimiento del impacto del programa. A lo largo del texto se analiza cuáles han sido los principales aciertos de la e-School para conseguir estabilidad educativa en la impartición de sus cursos, el freno a la deserción escolar y el uso de una plataforma educativa de vanguardia que combina diversas herramientas para aprovechar de manera eficaz los recursos tecnológicos de la información en universidades latinoamericanas. A la vez, se reflexiona en torno a los retos que han sido superados desde el inicio del programa y en su transición, así como los que se presentan en la actualidad, en especial la cuestión del financiamiento. Asimismo, se aborda la importancia de la formación de espacios para establecer el intercambio de experiencias educativas entre alumnos y profesores del programa que contribuyan a la construcción de una comunidad de coreanistas latinoamericanos a través de los talleres internacionales, lo que permitiría incrementar entre las universidades del continente el interés por los estudios de Corea en distintos ámbitos.
\end{abstract}

Palabras clave

Corea del Sur, América Latina, universidades, Programa e-School.

DOI: https://doi.org/10.36888/udual.universidades.2020.84.104

56 Universidades $\mid$ núm. 84, abril-junio $2020 \mid$ UDUAL 


\title{
Cooperação acadêmica entre Coréia do Sul e América Lati-
} na: o Programa e-School da Fundação da Coréia e o uso dos TIC para a internacionalização educativa

\section{Sumário}

Em esse artigo se apresenta a implementação, reestrutura e avaliação do Programa e-School para a América Latina, patrocinado pela Fundação da Coréia para impulsar os estudos da Ásia e da Coréia na região. É mencionada a mudança da sede da coordenação de Califórnia a Nuevo León como base fundamental para o aumento no impacto do programa. Ao longo do texto se analisa quais tem sido os principais acertos da e-School para alcançar a estabilidade educativa ao ministrar seus cursos, no freio na deserção escolar, e no uso duma plataforma educativa moderna que misture diferentes ferramentas para aproveitar eficazmente os recursos tecnológicos da informação nas universidades da América Latina. Além disso, se reflete em redor aos desafios que foram superados desde o início do programa e na sua transição, assim como também os que se apresentam na atualidade, principalmente a questão do financiamento. Da mesma forma, se aborda a importância da formação de espaços para estabelecer o intercâmbio de experiências educativas entre os professores e os alunos que contribuíram, através dos cursos internacionais, à construção de duma comunidade latino-americana interessada na Coréia, razão que permite o crescimento, nas universidades do continente, do interesse pelos estudos da Coréia em distintos âmbitos.

Palavras-chave

Coréia do Sul; América Latina; universidades; Programa e-School.

\section{Academic cooperation between South Korea and Latin Ame- rica: The Korea Foundation e-School Program and the use of TIC for the educative internationalisation}

\begin{abstract}
This article presents the implementation, restructure and evaluation of the Korea Foundation e-School Program for Latin America, created to encourage the Asian and Korean studies in the mentioned region. The research focuses on the change of the coordination's headquarters from California to Nuevo León as an essential movement of the program growing impact. Here is also an analysis about which are the e-School program good choices to acquire educative stability in the implement of courses, the brake of school dropout, and the use of an advance education platform that combines different tools to make the most of technological information resources in Latin American universities. At the same time, here is a reflection about challenges that have been accomplished from the beginning of the program, during in its transition, and those that are present, especially the financial. Additionally, this research refers to the importance of the forma-
\end{abstract}


tions of spaces to establish the exchange of educative experiences between the program students and teachers, that contribute to the construction of a Korean-study community in Latin America, through international workshops that will allow to increase the interest in the diverse fields of Korean research, between universities of the continent.

Keywords

South Korea, Latina America, University, e-School program

\section{Introducción}

La reciente y amplia presencia de Corea en el mundo ha detonado un incremento en el interés por conocer todo lo relacionado con ese país en áreas como política, economía y las relaciones internacionales, así como en su lengua, literatura, historia y cultura. Sin embargo, la mayoría de los países no tiene el personal especializado suficiente para satisfacer dicha demanda y Corea no cuenta con los recursos humanos y financieros para cubrirla a cabalidad. Para remediar la cuestión, la Fundación Corea (FC) creó en 2011 el Programa Global e-School, el cual es una estrategia de poder para la proyección internacional que consiste en la enseñanza y promoción del estudio de Corea en universidades públicas o privadas mediante cursos académicos virtuales en tiempo real a través de nuevas tecnologías de información y comunicación (TIC). Al igual que en los cursos tradicionales, los estudiantes tienen la responsabilidad de completar el programa temático y cumplir con las tareas y actividades establecidas por los instructores.

El Programa Global e-School fue establecido originalmente para cuatro regiones: Asia, Estados Unidos, Eurasia ${ }^{1}$ y América Latina. Esta división regional permite resolver problemas en cuanto a la diferencia de husos horarios y, en algunos casos, la conveniencia de impartir cursos en un idioma accesible. Los cursos ofrecidos para el continente asiático se imparten desde Corea, pero no hay una universidad que coordine de manera centralizada el programa como en el caso de Estados Unidos y América Latina, sino que participan varias, de manera independiente, y cada una se vincula y administra directamente con los grupos de las universidades asiáticas receptoras. $^{2}$

Por su parte, el e-School en Estados Unidos está conformado por un consorcio de universidades que conforman la llamada Big Ten Academic Alliance, que actualmente cuenta con 12 miembros. ${ }^{3}$ El consorcio se coordina desde el Centro Nam de Estudios Coreanos de la Universidad de Michigan. En este sistema, la universidad desde donde se imparte la clase funge como anfitriona para que otras universidades del consorcio reciban el curso, los cuales se definen según aquellos ya existentes dentro de la malla curricular de dichas universidades, se parte del principio de colaboración en el que las instituciones participantes comparten sus recursos. 
El Programa e-School para América Latina fue establecido bajo la coordinación del Centro de Estudios Coreanos de la Universidad de California, Los Ángeles (UCLA). Los cursos se impartían en inglés por profesores adscritos a esa universidad y otras también estadounidenses. En 2014 inició una nueva etapa al traspasar la coordinación al Centro de Estudios Asiáticos (CEA) de la Universidad Autónoma de Nuevo León (UANL) en México. Desarrollaremos con detalle ese proceso más adelante.

El enfoque de la FC respecto al alcance del Programa Global e-School en las diferentes sedes regionales y sus objetivos ha variado. En su etapa inicial, la prioridad del programa era más bien asegurar la calidad de los cursos y de sus estudiantes, por lo que se solicitaba que fueran grupos de licenciatura en semestres avanzados o bien estudiantes de posgrado. Posteriormente, la prioridad fue alentar la masificación de los cursos y sus receptores para optimizar los recursos, lo cual implicaba reducir los criterios de selección y aceptar el registro de grupos principalmente de licenciatura, sin importar el avance alcanzado por los alumnos en sus respectivos estudios universitarios. Tales objetivos se han ajustado constantemente en la búsqueda de un punto intermedio entre calidad y cantidad que fuera satisfactorio y estimulante para todas las partes, esto es, para la Fundación, las universidades coordinadoras y las receptoras.

Como incentivo adicional, el Programa e-School estableció un taller internacional anual en el que se invitaron a los estudiantes más destacados de cada curso. El taller tiene el propósito de profundizar en algunos de los temas abordados en las materias impartidas durante el año transcurrido y propiciar una interacción directa y cara a cara entre los estudiantes y los profesores. Como resultado, los estudiantes incrementaron su interés por el estudio de Corea y, para el caso de América Latina, ha facilitado la formación de una incipiente red de coreanistas jóvenes. El primer Taller Internacional para América Latina se llevó a cabo en la UCLA y a partir del segundo, en México.

Desde su establecimiento, el Programa para América Latina ha mostrado una notable capacidad de adaptación en el proceso enseñanza-aprendizaje y este trabajo da cuenta del proceso organizacional y operativo, su dinamismo e impacto en varios ámbitos, desde la transmisión y acumulación de conocimiento respecto al este de Asia, hasta la formación de una red académica netamente latinoamericana enfocada en el estudio de Corea. También revisa las diferentes problemáticas a las que se ha enfrentado y los principales desafíos que todavía tiene por resolver.

\section{Trayectoria del Programa e-School para América Latina}

El e-School para América Latina ha transitado por varias etapas marcadas por cambios en la coordinación, estrategias operativas y de administración. La primera etapa, que podríamos llamar de introducción, transcurrió de 2011 a 2014 y consistió en cursos impartidos por videoconferencia en inglés, pues estaba coordinado por la UCLA. Aunque la respuesta hacia el nuevo proyecto por parte de los colegas latinoamericanos fue aceptable y hasta cierto punto entusiasta, tuvo muchas dificultades ya que la FC establecía requisitos técnicos y cierta formalidad administrativa que hacían 
inviable la aceptación inmediata de las autoridades de las universidades potencialmente interesadas. Además, el idioma fue un factor importante ante un público estudiantil latinoamericano que todavía no cuenta con ese tipo de competencias. En septiembre de 2013, la UCLA anunció su intención de transferir la coordinación general del Programa e-School para América Latina y junto con la FC y el Comité de Desarrollo Curricular ${ }^{4}$ (CDC), creado en 2011, se optó por asentar la responsabilidad en el CEA-UANL pues contaba con un marco normativo y legal sólido para albergar el programa.

La segunda etapa que denominamos de transición comprendió de 2014 a 2017, cuando la coordinación fue finalmente transferida a la UANL y se llevaron a cabo cambios importantes. Por ejemplo, las clases comenzaron a ser impartidas en español -mayormente por profesores latinoamericanosy el uso de una plataforma de aulas virtuales de la empresa wizIQ cuya ventaja era que solo se requería que cada instructor y cada grupo tuvieran una computadora, un proyector estándar, altavoces y una buena señal de internet. En consecuencia, la demanda de receptores se incrementó enormemente, así como la oferta y diversificación de cursos con materias de ciencias sociales y humanidades. Con dicha ampliación, también aumentó el número de profesores que impartieron cursos completos o módulos en cursos compartidos. El reclutamiento inicial de instructores fue, principalmente, a partir de la red informal de algunos de los académicos más involucrados en el programa. El otro frente en expansión fue el de instituciones o personas interesadas en los cursos y también se llevó a cabo de manera personalizada a partir de las redes de colegas estudiosos de Asia.

En 2015, un semestre después de que la UANL asumiera la coordinación del programa, el CEA emprendió una estrategia de promoción más estructurada. Para ello se buscó a las cinco universidades más prestigiosas de algunos países latinoamericanos, preferentemente que tuvieran centros de estudios coreanos o asiáticos o, en su defecto, programas de grado o posgrado relacionados con Asia o, cuando menos, algún curso sobre esa región. Una vez identificadas, se estableció contacto con las autoridades de cada universidad y con las instancias relevantes para invitarlas a participar en el Programa e-School.

A partir de entonces, el número de cursos, universidades y estudiantes aumentó de forma creciente. En el semestre enero-junio de 2015 se ofrecieron 4 cursos en español en más de 30 universidades participantes de 6 países latinoamericanos y un total de 311 estudiantes. Además, en ese semestre se llevó a cabo el Cuarto Taller Internacional (el primero realizado por la UANL como sede del programa) al cual acudieron los estudiantes seleccionados por los profesores de cada curso durante el semestre enero-junio y agosto-diciembre de 2014.

En el semestre agosto-diciembre 2015 se incrementó aún más la cifra de estudiantes, alcanzando los 341 alumnos provenientes de 17 universidades diferentes. Los alumnos y universidades captados durante el semestre agosto-diciembre 2014 (el primero coordinado por la UANL) fue gracias a los contactos de universidades que tenían los profesores que impartieron los cursos, lo cual contrasta con la captación para el semestre agosto-diciembre 2015, después de realizar la estrategia de promoción mencionada anteriormente. 
En lo sucesivo, como mencionamos arriba, la expansión del programa obedeció a una estrategia de promoción intensiva cada vez más sistematizada, que apunta especialmente a los niveles más altos de autoridad en universidades que se mostraron interesadas o con las que se buscó un acercamiento por su importancia estratégica, prestigio y capacidad técnica. A diferencia de la etapa anterior, esta vez se procuró mayor formalidad con las universidades para lograr mayor estabilidad de grupos receptores y planificar mejor la oferta de cursos para cumplir con los tiempos administrativos de inscripción de las universidades. Mediante convenios interinstitucionales, el registro de estudiantes en cursos regulares que otorgaran créditos propiciaba mayor estabilidad y certidumbre de

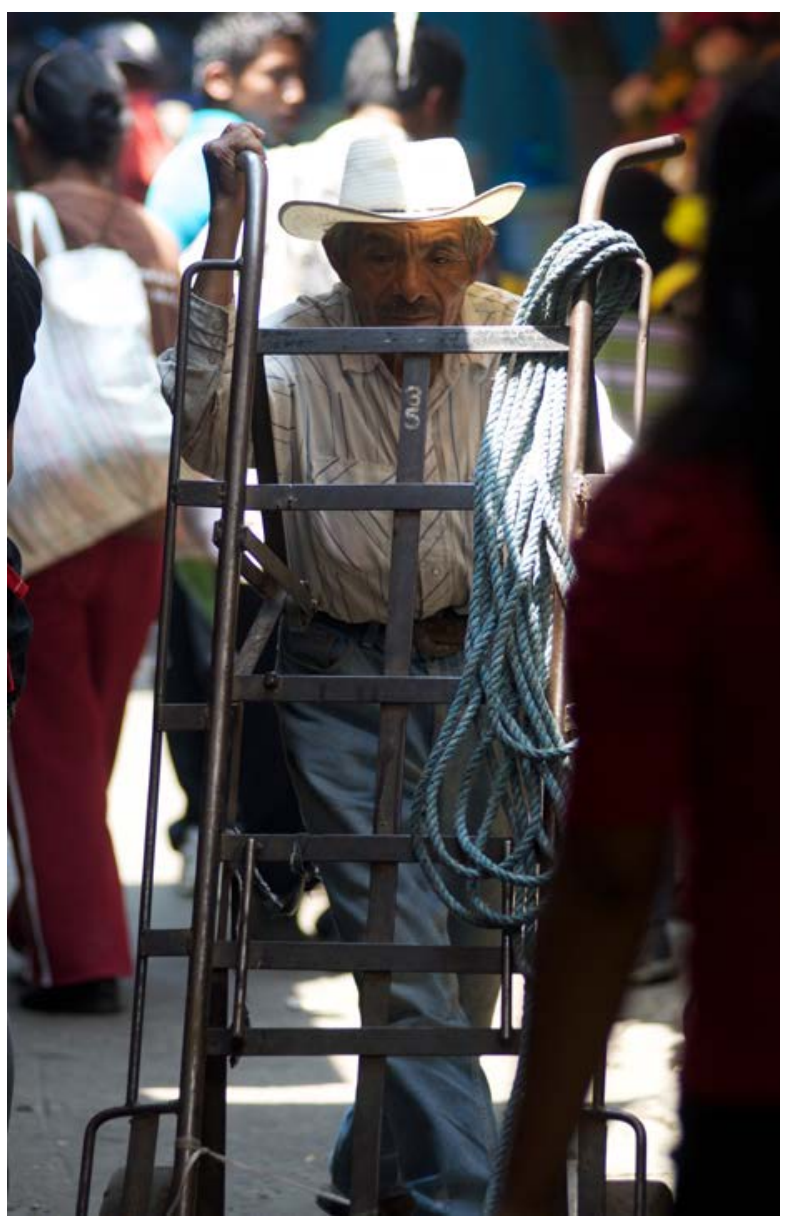
ambas partes. En consecuen-

cia, la eficiencia terminal del e-School creció significativamente, y eso contribuyó para obtener mayor confianza por parte de la Fundación Corea.

El semestre de primavera de 2016 fue muy importante porque más universidades comenzaron a incluir los cursos del programa en su malla curricular, es decir, que otorgaban créditos a los estudiantes que los concluían. En ese semestre se ofrecieron 4 cursos (3 en español y 1 en inglés), y se inscribieron 493 estudiantes en total. El crecimiento cuantitativo del Programa e-School causó gran impresión a los funcionarios de la Fundación Corea y a la comunidad académica que ha seguido de cerca este proceso.

Sin embargo, como era de esperarse, el crecimiento acelerado en el número de materias, profesores, universidades y estudiantes acarreó proporcionalmente una gran cantidad de complicaciones y problemas, sobre todo de índole administrativo, además de que la deserción fue mucho más notoria desde que el programa inició su franca expansión. Asimismo, aunque el crecimiento en cierto sentido era deseable, el financiamiento para mantener la expansión del programa, simplemente no era suficiente para cubrir las nuevas necesidades. Por lo anterior, se requirió una reingeniería operativa del programa. En este sentido, además de que se reformuló el papel de los coordinadores en cada sede, en 2017 el CDC aceptó la propuesta del CEA-UANL para establecer un consorcio de univer- 
sidades parecido al estadounidense, denominado G-13 e-School Program para América Latina. Las 13 universidades fueron seleccionadas según criterios como: haber establecido los cursos de e-School como cursos con créditos en su currícula, la cualificación de la persona asignada para ocupar la coordinación del programa en la universidad receptora, su compromiso con el programa y el apoyo institucional que tienen de sus universidades para impulsar los estudios coreanos. Con la formación del consorcio se establecieron nuevas y más eficientes formas de organizar el programa y la permanencia de las universidades participantes está condicionada al cumplimiento de los criterios señalados arriba. ${ }^{5}$

Con el establecimiento del G-13 el número de estudiantes inscritos disminuyó a 253, y el de países involucrados fueron 6 (341 estudiantes de 17 universidades y 5 países en el periodo agosto-diciembre de 2015). Esta reducción de universidades y estudiantes permitió reorientar el objetivo de trabajar por privilegiar la calidad sobre la cantidad.

Además del consorcio, se instauró un límite de 60 alumnos inscritos como regulares en cada curso, con lo cual se pudo asegurar un poco más el compromiso de los estudiantes que lograron un espacio y quienes, por lo general, son seleccionados por los coordinadores de cada universidad. Esta preselección es clave para contar con estudiantes realmente interesados en Corea y reducir lo más posible la deserción.

Las universidades que no pertenecen al G-13, pero que están interesadas en que sus estudiantes reciban cursos del e-School, o estén en espera de un espacio disponible por cancelación de alguna membresía, pueden hacerlo como universidades asociadas. Los estudiantes de estas últimas se inscriben como oyentes y no representan una carga de trabajo adicional para los instructores, ya que no tienen responsabilidad de asistir al número mínimo de clases ni entregar tareas y trabajos asignados, pero tampoco reciben un certificado al terminar el curso.

Como puede apreciarse, el Programa e-School de la Fundación Corea para América Latina se ha transformado bastante desde su inicio en cuanto al involucramiento institucional, la forma de impartir los cursos, la dinámica entre universidad sede y las universidades receptoras y el reclutamiento de profesores. Los cambios del programa en las etapas iniciales ocurrieron en reacción a las circunstancias cambiantes debido a los ajustes en las reglas, metas, expectativas y directrices establecidas por la Fundación Corea, así como por innovaciones técnicas y organizacionales propuestas y ejecutadas por el CEA-UANL, el CDC, los profesores del programa y por los colaboradores académicos de las universidades donde se reciben los cursos.

Cabe mencionar que el papel de la Fundación Corea ha sido muy positivo, no solo por el patrocinio financiero, sino por la autonomía académica y operativa otorgada al CEA-UANL y al CDC en cuanto al desarrollo de sus planes y proyectos. Es más, la Fundación ha sido lo suficientemente flexible para aceptar un gran número de innovaciones propuestas, aunque todavía hay cierta resistencia en cambiar algunas prácticas. 


\section{Características de los cursos}

A diferencia de los cursos en línea, el programa e-School para América Latina consiste en cursos sincrónicos, es decir, en tiempo real, que se imparten por medio de la plataforma de la empresa privada WizIQ. Con suficiente anticipación, el profesor o instructor entrega al personal del CEA-UANL el programa temático de la materia que impartirá, el cual es distribuido en las universidades receptoras participantes del e-School. Cada programa consiste en 48 horas de clase, dividido en 16 sesiones de tres horas cada una.

Los cursos pueden ser impartidos por una sola persona, pero también pueden estar diseñados en formato modular, con 2, 3 o hasta 4 instructores quienes se dividen los temas de acuerdo con sus respectivas áreas de especialización. En el caso de cursos modulares, el personal del CEA-UANL revisa el contenido temático y, en la medida de lo posible, busca coordinar la selección y secuencia de temas para optimizar el material y los recursos humanos en beneficio de los estudiantes, sin menoscabo de la libertad de cátedra.

A diferencia del modo anterior de videoconferencias a cargo de la UCLA -en el que se requería un costoso equipo generalmente ubicado en salones especiales-, en la etapa operativa a cargo de la UANL se utiliza una aplicación informática que genera un aula virtual. Existen en el mercado varias plataformas semejantes pero, desde su inicio, el Programa e-School para América Latina contrató una cuenta de wizIQ, la cual fue aceptada por el CDC en su reunión de 2014 y posteriormente por la Fundación Corea por parecer lo suficientemente amigable, aún para profesores no expertos en las TIC. Esta plataforma, en particular, no requiere de descargar e instalar alguna aplicación en la computadora del profesor, estudiante o grupo receptor. Cada sesión se programa de manera anticipada y se genera una liga específica que se activa el día y la hora establecida. Cada profesor o instructor es responsable de proporcionar al CEA-UANL la presentación y el material de lectura que requiera.

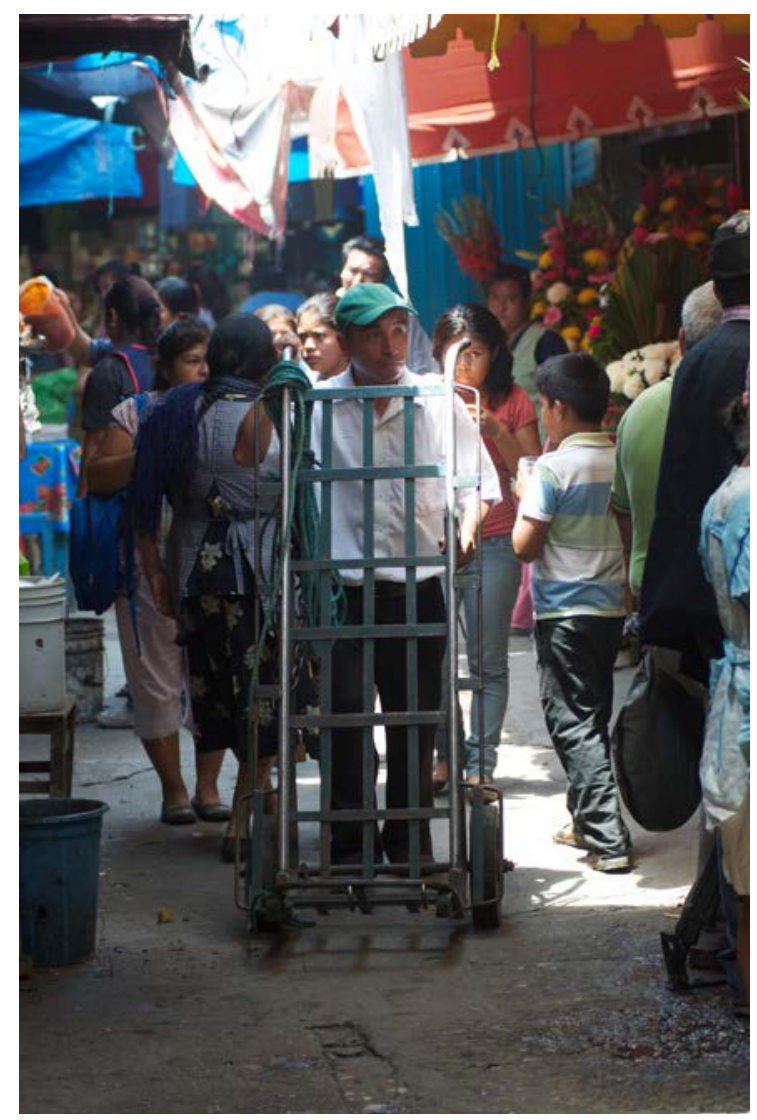


El video del instructor aparece automáticamente en un recuadro preestablecido y los coordinadores y estudiantes pueden interactuar activamente durante la sesión por medio de la herramienta de conversación por escrito (chat). Los coordinadores y estudiantes receptores también pueden escribir en el "pizarrón", usar el micrófono, e incluso transmitir video, aunque deben tener permiso concedido por parte del instructor titular. Cada sesión queda grabada en la cuenta adquirida por el CEA-UANL.

Finalmente, la plataforma WizIQ es compatible con otras usadas en MOOC como Moodle, Blackboard, Joomla, Sakai, etcétera; lo cual incrementa de manera importante las herramientas para una experiencia integral de aprendizaje. Además, los profesores pueden emplear otras herramientas informáticas gratuitas para crear dinámicas paralelas y fortalecer la comunicación, el intercambio de material y la colaboración en línea, como Google Drive, Dropbox, Survey Monkey, Flipgrid, Poll Everywhere, entre otras. De esta manera, salvo por la falta de interacción física de la forma de educación tradicional, la experiencia de aulas y cursos virtuales del e-School puede resultar estimulante.

\section{Actividades adicionales}

Además de los cursos, el Programa e-School desarrolla dos actividades anuales: el Taller Internacional y los cursos intensivos. El taller o Workshop se lleva a cabo durante dos días, generalmente en la universidad coordinadora del e-School. Como mencionamos arriba, el primero fue en la UCLA (2012) y, por cuestión de costos y facilidad logística, el segundo y tercero en la UNAM (2013 y 2014), aun cuando la coordinación general seguía estando a cargo de la UCLA. A partir de 2015 la sede de los talleres ha sido la UANL. A cada taller se invita, con todos los gastos pagados, a los estudiantes más destacados durante el año escolar inmediato anterior, algunos profesores que impartieron un curso durante ese periodo y una selección de coordinadores de grupo. También se invita a profesores destacados en alguna temática particular de Corea para impartir una conferencia magistral y colaborar en los trabajos del taller.

El taller ha tenido modificaciones importantes desde sus primeras versiones. En todos los casos se han impartido conferencias magistrales o paneles de discusión de profesores, en un formato tradicional de conferencia y un tiempo final para preguntas y respuestas. En ediciones más recientes se ha buscado la participación más activa de los estudiantes, de tal manera que puedan ser escuchados y, sobre todo, discutir a detalle los temas que a ellos les interesan más. Como ocurrió con los cursos, la experiencia y el aprendizaje mediante prueba y error han transformado la práctica del taller para convertirse en un ejercicio de intercambio colectivo de conocimientos mucho más interesante, dinámico y provechoso.

El taller ha servido como uno de los incentivos más importantes para muchos alumnos, quienes no solo hacen un esfuerzo especial para concluir los cursos, sino que saben que de hacerlo muy bien pueden ser recomendados por sus coordinadores y los profesores, quienes toman en cuenta la asistencia a cada sesión, la asiduidad y pertinencia de sus participaciones durante el semestre, así como la calidad de sus trabajos. Por supuesto, aque- 
llos que asisten al taller reciben un reconocimiento especial con un certificado impreso y firmado por autoridades universitarias y de la Fundación Corea. Al final la convivencia y el intercambio de ideas, experiencias y emociones constituyen la base para la formación de una comunidad de coreanistas jóvenes que, en muchos casos, los ha encauzado a continuar el estudio de Corea en sus respectivos países y mantener el contacto con sus compañeros, para así formar una red que se espera rinda frutos a largo plazo.

Paralelamente al Programa e-School, en 2015 la Fundación Corea estableció un programa de cursos intensivos presenciales orientados a las diferentes universidades latinoamericanas participantes, los cuales podrían ser impartidos en inglés o español. A pesar de que los destinatarios eran universidades latinoamericanas, los cursos eran coordinados directamente por el Centro de Estudios Coreanos de la UCLA, por lo que se impartían en inglés y únicamente por profesores de esta institución. Los cursos consistían en sesiones de 4 horas por día durante 2 semanas, para así sumar 40 horas. Los estudiantes que finalizaban y aprobaban el curso recibían un certificado de la UCLA. Dado que el programa solo contaba con 2 cursos intensivos por año, se intentaba que se impartiera uno durante el semestre de primavera y el otro en el de otoño.

\section{Problemáticas institucionales, operativas, técnicas y curriculares}

Aun cuando el Programa e-School de la Fundación Corea para América Latina ha sido exitoso y es reconocido por varias universidades latinoamericanas como una oportunidad de internacionalización educativa, se trata de un proyecto que ha tenido que superar muchos obstáculos de diversa índole. En esta sección destacamos algunas problemáticas que han sido resueltas total o parcialmente.

\section{Disposición de autoridades}

Es un hecho que Corea es un país cada vez más conocido y que el interés por saber más sobre su cultura, su sociedad, su política y su economía es un factor determinante en la popularidad de los cursos. A pesar del creciente interés en Corea y la promoción realizada por el CEA-UANL, la gestión para la apertura de cursos tan específicos en el mapa curricular de algunas carreras universitarias requiere una gran labor de convencimiento, que no necesariamente se basa en la importancia de los estudios de área per se, sino en factores más pragmáticos y normativos. Por lo general, la respuesta de las autoridades suele ser positiva y entusiasta, especialmente de universidades pequeñas que bus- 
can vínculos internacionales para sus programas de educación continua y a distancia, pues esto incrementa gradualmente su relevancia para afianzar la imagen institucional y, además, porque suele formar parte de los nuevos criterios normativos de evaluación y acreditación por parte de las autoridades educativas de los países latinoamericanos. Eso explica en parte el interés de varias universidades latinoamericanas receptoras por firmar convenios de cooperación interinstitucional e incluir cursos internacionales del e-School en sus programas.

\section{Problemas operativos de la expansión del Programa e-School}

Mencionamos anteriormente que las estrategias de promoción por parte del CEA-UANL fueron exitosas en cuanto a la expansión cuantitativa del Programa e-School para América Latina. Al inicio, la tarea consistió en aumentar el número de estudiantes sin restricciones ni filtro alguno, por lo que cualquier persona podía inscribirse de manera individual o formar un grupo de alumnos entusiastas sin siquiera tener un coordinador. A pesar del creciente número de alumnos, el personal del CEA-UANL y el CDC reconocieron las deficiencias de este proceso, especialmente en cuanto a la deserción que se hacía notar hacia la mitad de cada semestre.

Por otra parte, el gran número de universidades y alumnos inevitablemente trajo consigo problemas de administración y operación al personal del CEA-UANL, pues con un reducido staff y un presupuesto modesto e inflexible tenían que resolver situaciones que se multiplicaban exponencialmente cada semestre. Esto afectó también a los instructores de los cursos, ya que, además de su asignación normal de docencia, aumentó su carga laboral al tener que atender casi directamente a varias docenas de estudiantes de toda latinoamérica, provenientes de diversas disciplinas, tanto en etapas iniciales como finales de formación universitaria y de bagajes educativos muy dispares, esto aunado a la dificultad intrínseca de la comunicación a distancia y la incapacidad de conocer a detalle las circunstancias de cada alumno y alumna por el carácter impersonal del aula virtual.

En consecuencia, hubo numerosos casos de tensión porque el CEA-UANL simplemente no podía satisfacer los cuantiosos requerimientos de toda índole por parte de alumnos que demandaban atención detallada a sus respectivos asuntos. En cuanto a los profesores e instructores del programa, también tuvieron que idearse formas de simplificar la carga de trabajo para minimizar el desgaste provocado por el gran número de alumnos que debían atender. Se formularon respuestas estandarizadas como contratos e instructivos pormenorizados a profesores, coordinadores y autoridades que, en su mayoría, lograban satisfacer las solicitudes recibidas. Empero, la calidad de la atención individual ciertamente sufrió una merma durante el 2015 
y hasta mediados de 2016, debido a la masificación del programa en esa etapa. En pocas palabras, la expansión del e-School para América Latina provocó que la atención del profesorado se dispersara y se enfrentó al clásico dilema de optar entre cantidad y calidad.

Con el reordenamiento del programa entre 2016 y 2017 y la conformación del consorcio o G-13, la respuesta al dilema se inclinó por favorecer la calidad del programa en general, por lo que los problemas mencionados arriba se redujeron sensiblemente. Para empezar, el número de universidades receptoras se contrajo a una cantidad fija, lo cual ha dado mayor estabilidad y fluidez a la comunicación y se puede esperar mayor compromiso institucional.

Al establecer cupos o lugares disponibles a cada universidad y la fijación de un número máximo de estudiantes por curso (60), también se logró reducir la carga a los instructores y mejorar su desempeño en cuanto a la atención y evaluación de estudiantes. Asimismo, se confirió un papel más formal y activo a los coordinadores en las diferentes sedes en cuanto a responsabilidades de supervisión, monitoreo y seguimiento, acompañado de un estipendio superior al recibido anteriormente, el cual está sujeto al cumplimiento de sus responsabilidades. Esto fue posible precisamente gracias al nuevo orden del consorcio, ya que el presupuesto asignado para el rubro de coordinadores no aumentó, sino que su empleo se hizo más efectivo. El renovado papel de los coordinadores también contribuyó a una mejor retroalimentación entre los grupos y los profesores, gracias al contacto directo entre ellos y el llenado de un formulario semanal que debe enviarse al personal del CEA-UANL y a los profesores de la cátedra.

Otro factor relevante en el proceso de consolidación fue la estabilidad en la oferta de cursos. Después de dos años de aprendizaje en el reclutamiento de profesores e invertir tiempo en una capacitación técnica adecuada, el e-School ha establecido una plantilla docente permanente y estable con un profesorado comprometido y de una alta calidad académica. Los docentes que forman la base del programa son aquellos que, según las encuestas y las observaciones del equipo del CEA-UANL en consulta permanente con miembros del CDC, lograron combinar un buen contenido académico con una dinámica acorde a las necesidades y expectativas de los estudiantes, que demostraron comodidad el uso del software y lo utilizaron a cabalidad. Además, el personal del CEA ha establecido lineamientos estandarizados escritos con gran claridad y detalle, los cuales son entregados a los profesores con mucha antelación, de tal forma que desde el principio conocen sus responsabilidades y pueden decidir si están dispuestos a comprometerse con el programa.

Gracias a que el programa cuenta ahora con un grupo estable de docentes, es posible programar los cursos con suficiente 
anticipación para que las universidades a su vez los puedan registrar en sus plantillas o mallas curriculares y ofertarlos entre sus estudiantes. A diferencia de los primeros dos años, ahora existe completa certidumbre en la programación anual y semestral de las universidades del G-13.

\section{Retos técnicos y curriculares en las universidades receptoras}

La ventaja del software WizIQ que se emplea en el e-School para América Latina es que brinda flexibilidad en cuestión del espacio y los requerimientos tecnológicos de las universidades receptoras y los profesores. Con programas informáticos de aulas virtuales, la sesión puede ocurrir en cualquier salón que tenga acceso a una buena señal de internet y un proyector. Es más, los profesores también pueden llevar a cabo su clase desde cualquier parte del mundo. Entre los problemas más comunes encontramos la insuficiente amplitud de la banda o problemas de conectividad en la red. Este tipo de cuestiones técnicas son resueltas por las propias universidades y no compete al CEA-UANL intervenir al respecto. Lo que sí puede hacer es considerar la capacidad e infraestructura tecnológica como un elemento central en el proceso de selección del G-13.

En cuanto al perfil curricular de las universidades latinoamericanas, ha sido extremadamente difícil encontrar instituciones de educación superior que tengan interés en Asia, no se diga en un tópico tan específico como el estudio de Corea. Paradójicamente, aunque en todo el mundo se reconoce la importancia de Asia y hasta se dice que el futuro y el motor económico global se encuentra en esa región, pocos son los esfuerzos y proyectos concretos que llenan ese vacío en los programas curriculares de educación superior en América Latina. Para un programa académico como el e-School esto es fundamental, pues los cursos están destinados a proveer de conocimientos y análisis avanzados sobre Corea y Asia. Sin embargo, debido a la diversidad de estudiantes y las brechas en cuanto a su conocimiento previo sobre historia y economía política no solo de Corea, sino de Asia entera, conducen a que los cursos conserven un carácter introductorio y general, por lo que la aspiración de profundizar en los temas debe ser moderada.

Salvo algunas excepciones, las universidades latinoamericanas no están haciendo ajustes significativos en sus programas académicos para colocar Asia en el mismo plano de relevancia que Estados Unidos o Europa. ${ }^{6}$ En consecuencia, los estudiantes que toman los curos del e-School no solo carecen de un conocimiento básico de la región asiática, sino que los cursos son los únicos que existen sobre Asia en su mapa curricular. Lo anterior puede tener cierto mérito, pues confiere una gran responsabilidad al programa. Sin embargo, el oasis que representa el e-School en el desierto académico latinoamericano es insuficiente para consolidar los estudios de Corea y Asia y aspirar a la formación de especialistas que avancen el estudio a niveles equiparables a los de Estados Unidos y Europa. Para ello se requiere una planeación estratégica por parte de las universidades y no solo como iniciativas aisladas de algunos de académicos quienes, por muy diversos motivos, razones y circunstancias, se han formado en disciplinas afines y han optado por desarrollar estudios avanzados sobre Asia y/o en Asia. Varios de esos académicos han sido claves para posicionar el Pro- 
grama e-School en sus respectivas instituciones, pero son una minoría, no son todos coreanistas y no es posible contar con ellos de manera estable.

Lo anterior quiere decir que, aunque el Programa e-School lleva a cabo una labor académica extraordinaria en el sentido de ir más allá de sus objetivos originales (la promoción del estudio de Corea en América Latina), es preciso que las instituciones de educación superior en la región impulsen aún más los estudios de Asia en general. Ciertamente el impulso debe ocurrir desde los niveles más altos de autoridad universitaria, pero también de los niveles medios y de la demanda de los estudiantes, pues se requiere la formación de sinergias hacia la formación de recursos humanos especializados en aquella región. Las autoridades pueden detonar la demanda mediante convenios de intercambio, promoción de becas y establecimiento de departamen-

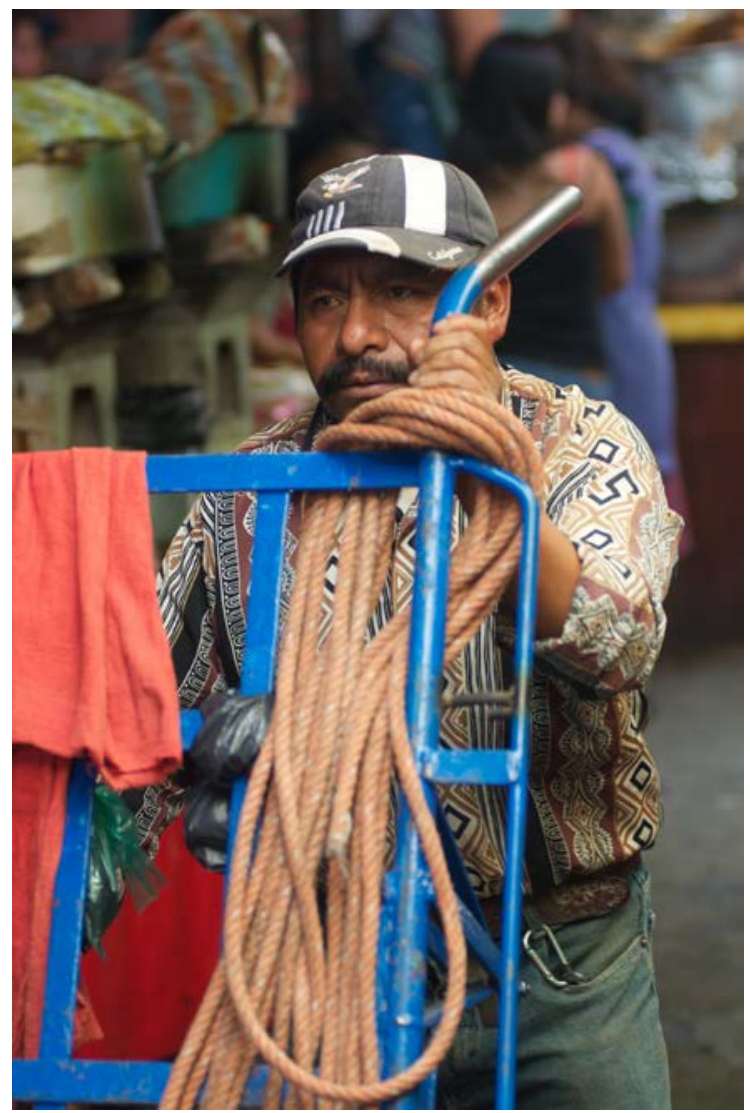
tos académicos enfocados en Asia, preferentemente con una orientación multidisciplinaria. También deberían incluirse más materias sobre Asia o en las que Asia aparezca en el programa temático. Ese tipo de acciones son fundamentales para la formación gradual y constante de recursos humanos que, eventualmente, conformen la masa crítica necesaria para ampliar y profundizar la transmisión, generación y acumulación de conocimiento sobre la región.

\section{Retos en el seguimiento, estabilidad y autonomía financiera}

Finalmente, el e-School todavía tiene que idear e instrumentar mecanismos de seguimiento de sus actividades, sus logros y, más importante, los de los estudiantes. Es muy difícil seguir la pista de tantos alumnos, además de costoso y quizás innecesario. El Taller Internacional que se lleva a cabo anualmente en Monterrey puede ser un medio para la integración, la convivencia y la gestación de un sentido de pertenencia a la comunidad latinoamericana de coreanistas, y también como preámbulo para la formación de algún mecanismo de seguimiento a los alumnos destacados egresados del e-School.

Se sabe que, a raíz del Taller Internacional, otros estudiantes han seguido en contacto por iniciativa propia y resulta gratificante saber que varios de ellos han encontrado en el estudio de Asia, y Corea en particular, una línea de estudio, con lo cual se puede dar continuidad. Otros han decidido tomar 
varios cursos del e-School para así tener una visión más completa de Corea y la región asiática, con el firme propósito de formarse en estudios de esa región. Podría ser conveniente establecer algún sistema informático para identificar a los estudiantes que han tomado más de un curso y dar seguimiento particular a su trayectoria. El CEA-UANL, además, ha desarrollado una amplia red interinstitucional y de titulación conjunta que puede ser un incentivo para que ese tipo de ex alumnos del programa continúen sus estudios en programas afines.

Por otra parte, una cuestión central en la estabilidad del Programa e-School para América Latina es, sin duda, el financiamiento. Nada de lo logrado hasta ahora habría sido posible solo con el entusiasmo, esmero e interés de aquellos que han dedicado su tiempo y energía a promover el programa y los cursos, ni de las decenas de profesores que los han impartido, ni la participación diligente y vital de los coordinadores; tampoco sería posible la realización del Taller Internacional o los cursos intensivos. Todo eso requiere financiamiento y éste ha sido en su gran mayoría provisto por la Fundación Corea.

El Programa e-School para América Latina todavía no está en condiciones de seguir sus tareas de manera autosuficiente. Para ello, no solo el programa debe estar consolidado en sus funciones operativas y en la estructura como podríamos decir que está en este momento, sino que se requiere que la demanda en estudios coreanos sea lo suficientemente firme en la región como para poder recibir recursos financieros de los receptores y que el programa sea costeable en caso de que alguna vez no se cuente con el auspicio de la Fundación Corea. Todavía no se ha realizado ningún estudio de mercado para determinar en qué punto se encuentra la demanda de estudios coreanos en América Latina, pero podría inferirse que las universidades todavía estarían más dispuestas a invertir en estudios de China o Japón o bien en un enfoque regional, en lugar de uno tan particular como el de Corea. Eso quiere decir que la promoción debe mantenerse y desarrollarse de manera constante, sobre todo porque China y Japón también invierten en la internacionalización de su imagen. Asimismo, en el mercado de cursos en línea existen un gran número de cursos gratuitos, varios de ellos de universidades muy prestigiosas. Como en otras áreas de la política y la economía internacional, Corea enfrenta una competencia formidable y el proyecto educativo tipo 
e-School, como medio para ejercer el poder suave, no es la excepción. Por lo tanto, la Fundación Corea todavía puede encontrar útil sostener el programa con el financiamiento correspondiente.

Finalmente, un punto importante para que la autonomía financiera no sea por ahora una prioridad es que la Fundación Corea ha confiado en los criterios del CEA-UANL, así como del CDC, para la selección de materias y docentes que las imparten. El respeto mostrado por la Fundación en el trabajo colegiado de los académicos latinoamericanos contribuye a una actitud recíproca por parte de la comunidad y a la entrega de resultados deseados, a veces más allá de las expectativas.

\section{Conclusiones}

El Programa e-School de la Fundación Corea para América Latina es relativamente nuevo y ha transitado por varias etapas. Los cambios han sido respuestas a circunstancias diversas y en diferentes niveles, pero también a la planificación consciente y metódica de sus coordinadores. Tanto las decisiones de la Fundación Corea en cuanto a sus preferencias (por ejemplo, cantidad frente a calidad, el formato, etcétera), la disposición de coordinar el programa por parte del Centro de Estudios Coreanos de la UCLA, y el relevo del CEA-UANL en la operación de éste han sido factores que impactaron su estructura y características. El liderazgo y visión de los coordinadores en la UCLA y en la UANL también han sido determinantes, en especial su capacidad de convocatoria y su habilidad para trabajar de manera colegiada y, sobre todo, transparente.

Puede decirse que el e-School también ha evolucionado a partir de la experiencia de sus coordinadores, sus equipos de trabajo y de los profesores que han colaborado. Todo se ha aprendido en el camino, pero aun así, la expansión pudo ser administrada y la consolidación está en marcha con un esquema asequible y controlable. Sin embargo, todavía no queda claro cómo es que funciona el programa en otras regiones, lo cual sería de gran ayuda para mejorarlo en América Latina y, posiblemente, la experiencia latinoamericana pueda contribuir en innovaciones positivas para el Global e-School en general.

Con la reorganización del e-School para América Latina en el G-13, podría decirse que el programa llegó a un punto de consolidación que le permite al CEA-UANL tener más control sobre los recursos y hacer un uso eficiente de ellos. Además, alivia la carga al docente, lo cual le permite una mayor concentración en la preparación de contenidos y dinámicas de interacción para cada sesión. Finalmente, los estudiantes se sienten más motivados, pues la mayoría de los cursos son válidos curricularmente, obtienen créditos requeridos para su titulación y reciben una gran experiencia educativa que puede concluir en un viaje al Taller Internacional. 


\section{Retos del programa y oportunidades de réplica}

El Programa e-School ha sido exitoso en muchos sentidos, tanto en la etapa de la coordinación de la UCLA como de la UANL. El principal logro ha sido responder al objetivo central de la Fundación Corea que es promover y difundir extensamente el estudio de Corea. Esta tarea, vale recalcar, se ha logrado con un amplio espacio de autonomía por parte de la Fundación Corea en cuanto a la utilización de las TIC y en la selección de cursos, contenidos y cuerpo docente. También ha sido exitoso en cuanto a producir cursos con una calidad aceptable no obstante los obstáculos técnicos, tecnológicos y la gran diversidad de bagajes educativos existentes en las universidades de América Latina. También es cierto que todavía existe un buen número de retos por zanjar y en este artículo pudimos identificar algunos de ellos, especialmente ante la posibilidad de que esta experiencia pueda ser útil a otras iniciativas para la internacionalización educativa.

\section{Notas}

1. El e-School para Eurasia se lanzó en 2012 dentro de la Universidad Central Europea (Central European University-CEU) en Hungría, y contaba con 8 universidades, pero actualmente se encuentra suspendido.

2 Existen muchas universidades en Asia que cuentan con programas de licenciatura y posgrado especializados en Corea, por lo cual los estudiantes pueden recibir los cursos en coreano y en inglés y obtener créditos completos porque suelen estar registrados formalmente en el mapa curricular.

3. Las universidades participantes del consorcio son: Universidad de Indiana, Universidad Estatal de Michigan, Universidad Estatal de Ohio, Universidad de Iowa, Universidad Estatal de Pennsylvania, Universidad Purdue, New Brunswick, Universidad de Illinois en Urbana-Champaign, Universidad de Maryland, Universidad de Michigan, Universidad de Minnesota y Madison.

4. El CDC tenía la función de un cuerpo colegiado que aportaría una visión integral al programa y donde se discutirían asuntos académicos respecto a la selección de materias de acuerdo con las necesidades e intereses de las universidades involucradas y evaluar su desempeño general. La formación del Comité buscaba la representatividad geográfica e institucional de la academia latinoamericana con potencial para desarrollar estudios sobre Corea en la región.

5. Otros requisitos de permanencia que deben cumplirse son: entregar reportes semanales a la sede del programa (la UANL) para cada curso que estén recibiendo, registrar a un determinado número de estudiantes por semestre, que el $70 \%$ de sus estudiantes registrados aprueben el curso que tomaron, entre otros.

6. La universidad EAFIT en Colombia, la Universidad Central de Chile y la Universidad de Costa Rica, pueden contarse en ese pequeño grupo de instituciones de educación superior que cuentan con programas especializados en Asia. En el caso de la Universidad Central de Chile y la Universidad de Costa Rica, se han abierto programas especializados en Corea, pero son de muy reciente creación y están apenas en proceso de germinación.

72 Universidades $\mid$ núm. 84, abril-junio 2020 $\mid$ UDUAL $\mid$ DOI:https://doi.org/10.36888/udual.universidades.2020.84.104 Cooperación académica entre Corea del Sur y América Latina: el Programa e-School de la Fundación Corea y el uso de TIC para la internacionalización educativa Juan Felipe López Aymes, Renato Balderrama Santander y Ana Karen Velázquez 
$40 x$ tos.

$\frac{1}{6-2}+2$

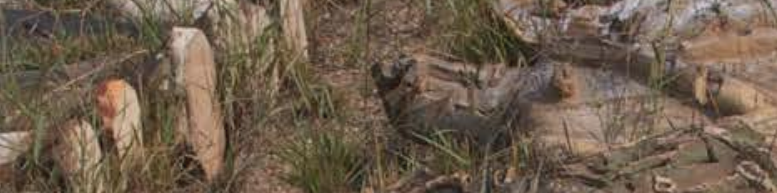

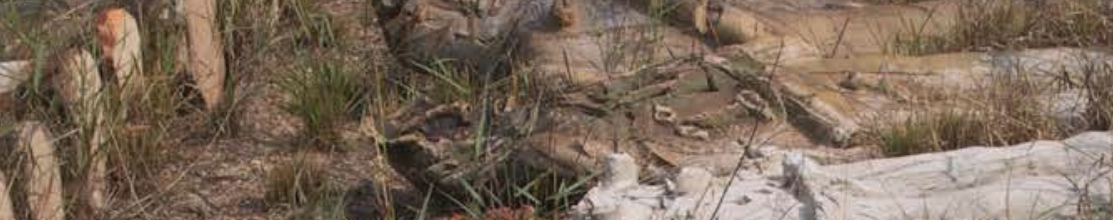

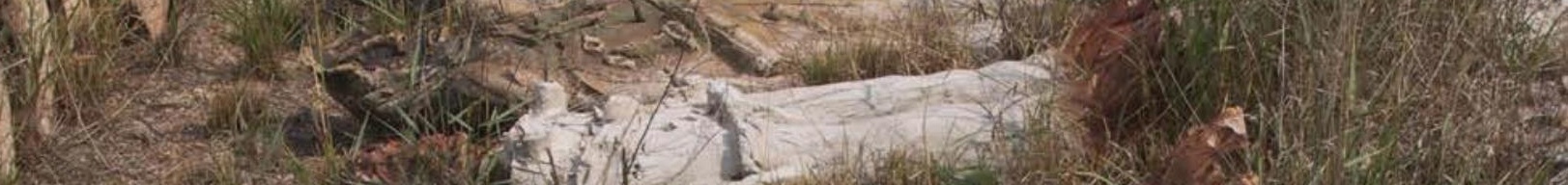

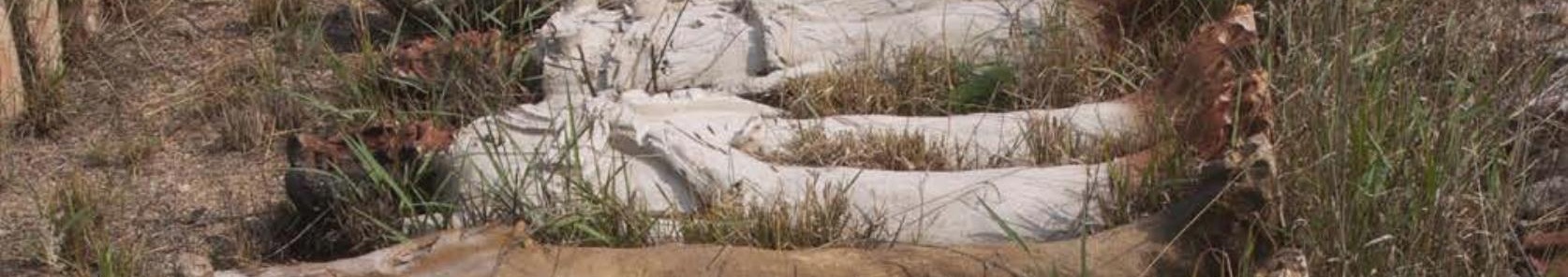

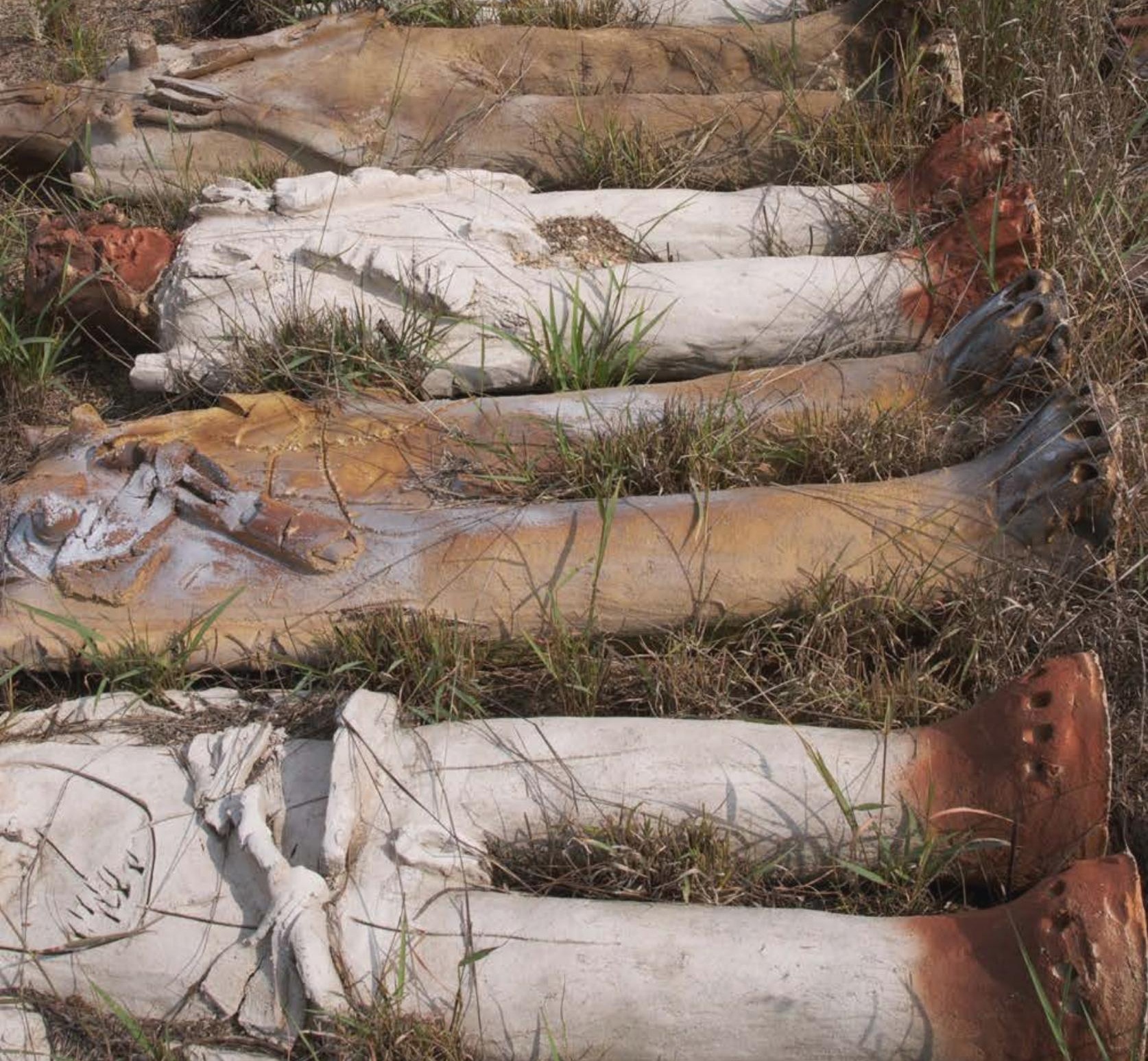

